\title{
An Adaptive Parallel Interference Mitigation Technique using Artificial Neural Network in Complementary Coded MC-CDMA
}

\author{
Supreeti Bal ${ }^{1}$, Mritunjay Kumar Rai ${ }^{2}$, Hye-jin $\mathrm{Kim}^{3}$ and Rahul Saha ${ }^{4 *}$ \\ ${ }^{1,2}$ Department of Electronic and Communication Engineering, \\ Lovely Professional University, India \\ ${ }^{3}$ Business Administration Research Institute, Sungshin W. University, \\ 2 Bomun-ro 34da gil, Seongbuk-gu, Seoul, Republic of Korea \\ ${ }^{4}$ Department of Computer Science and Engineering, \\ Lovely Professional University, India \\ *rsahaaot@gmail.com
}

\begin{abstract}
In Multi-Carrier Code Division Multiple Access (MC-CDMA) method, multiuser detection in fading channel scenario is a major challenge. The scope of this work is to design a multiuser detection technique using Parallel Interference Cancellation (PIC) with Artificial Neural Network (ANN) for multiple access interference mitigation. MCCDMA with equalization has become popular because it is able to offer better performance than conventional system in frequency selective fading channel. In this paper, performance of PIC with maximum likelihood (ML) equalization is analyzed. For optimizing the output of PIC and reducing the Bit Error Rate (BER) an artificial neural network is used. Feed forward multilayer perceptron model is applied in our proposed method. Simulation results prove that proposed combination of Artificial Neural Network and Parallel Interference Cancellation technique has better multi access interference (MAI) mitigation strength than PIC with ML equalizer.
\end{abstract}

Keyword: Artificial, interference, CDMA, multi-carrier, multiple access, likelihood, neural, perceptron, parallel, cancellation, mitigation

\section{Introduction}

In today's mobile telecom era users demand higher speed of data transfer along with voice; to match such demands larger bandwidth is required to accommodate voice and data. The different impairments affecting the capacity and high data rate include delay spread, multipath fading and Multiple Access Interference (MAI). In multi access techniques many users can share the same bandwidth efficiently. One of the multi access technique known as Code Division Multi Access (CDMA) is more popular in comparison to other techniques for its better performance in terms of capacity, more number of users, system security and coverage [1]. In CDMA, every user uses a different code to use same bandwidth. MAI and multi path interference, limit the capacity of wireless systems. To avoid MAI, the codes used in CDMA systems must have low or zero cross correlation. Orthogonal Complementary Code (OCC) is also introduced which have zero auto correlation and absolute zero cross-correlation. OCC performs much better in multiple users' scenario [2].

An interference free multi carrier transmission is possible by choosing mutually orthogonal sub carriers. But in the wireless scenario it is difficult to maintain

Received (March 16, 2018), Review Result (June 9, 2018), Accepted (June 14, 2018)

* Corresponding Author 
orthogonality of the sub carriers. Hence, at the receiver side multiple access interference occurs which limits the capacity of MC-CDMA systems [3]. The MC-CDMA transmitter spreads the data in the frequency domain over different subcarriers using a given spreading code. For downlink mobile radio communication channel, the application of orthogonal codes such as complementary codes ensure the absence of Multiple Access Interference (MAI) in a Gaussian channel. However, in a frequency selective fading channel, all the subcarriers have different amplitude levels and different phase shifts which results in a loss of the orthogonality among users and therefore generates MAI [4]. After applying Fast Fourier Transformation (FFT) the received sequence must be equalized by using one equalizer per subcarrier. To combat the MAI, various basic equalization techniques such as Maximum Likelihood (ML), Maximum Ratio Combining (MRC), Equal Gain Combining (EGC), Orthogonal Restoring Combining (ORC) or Minimum Mean Square Error (MMSE) may be used [5]. In this work Maximum Likelihood Equalization is applied independently on each subcarrier. ML equalizer provides near optimum value and has high error detection and correction ability. It provides better recovery of data than MMSE and Zero Forcing (ZF) equalizer [6].

MAI can occur due to any one user is insignificant but as the number of interfering users increases multi access interference becomes significant [7]. A conventional detector does not consider existence of MAI. Later, Single User Detection (SUD) techniques are evolved in which each user is detected individually irrespective of other users [8]. However, due to interference amongst users, Multi User Detection (MUD) is a better technique. In MUD, multiple users' information is used jointly for improved detection of individual user. The main motive of MUD is to search the ways to process output of matched filter so that the transmitted bit can be detected precisely. MUD technique can be divided into two categories such as optimal and sub optimal. In optimal decoding technique comparison of the received sequence with all the possible code sequences is performed. Maximum likelihood (ML) performance can be achieved. In ML detection when the number of users increases substantially system becomes more complex [9]. This reason led to development of sub optimum detectors. There are two types of sub optimal techniques, linear detectors and nonlinear detector. Linear detectors are further divided into Minimum Mean Squared Error and de-correlating detector. Nonlinear detector can be classified as Successive Interference Cancellation (SIC) and Parallel Interference Cancellation (PIC). SIC is applied in multiple stages in which it cancels serially the output of matched filter. PIC cancels all the estimated signals of users from the combined signal received [10].PIC also works in multistage like SIC but residual error obtained in each stage is subtracted in next stage and this process continues till there is no error found [11].

In this paper, the focus is on enhancing the output of complementary coded MCCDMA system by decreasing MAI under nakagami fading channels. PIC with ML equalizer is proposed for obtaining interference free performance in complementary coded MC-CDMA. The performance of BER can be improved by using multilayer perceptron model which has three layers such as input layer, a middle layer known as hidden layer and last as output layer. A Neural Network model is made up of neurons which are interconnected. These neurons pass information and exchange message to each other. Particular numerical weights are assigned to each connection which can be changed to get the desired output depending upon the application [12][13]. The multilayer perceptron model is described in Figure 1. Learning ability is a key component of neural networks. So, with help of learning algorithm a neural network can be trained, modify its weight for a particular output. This goal of this work is to provide an efficient MUD technique using combination of PIC and Artificial Neural Network. 


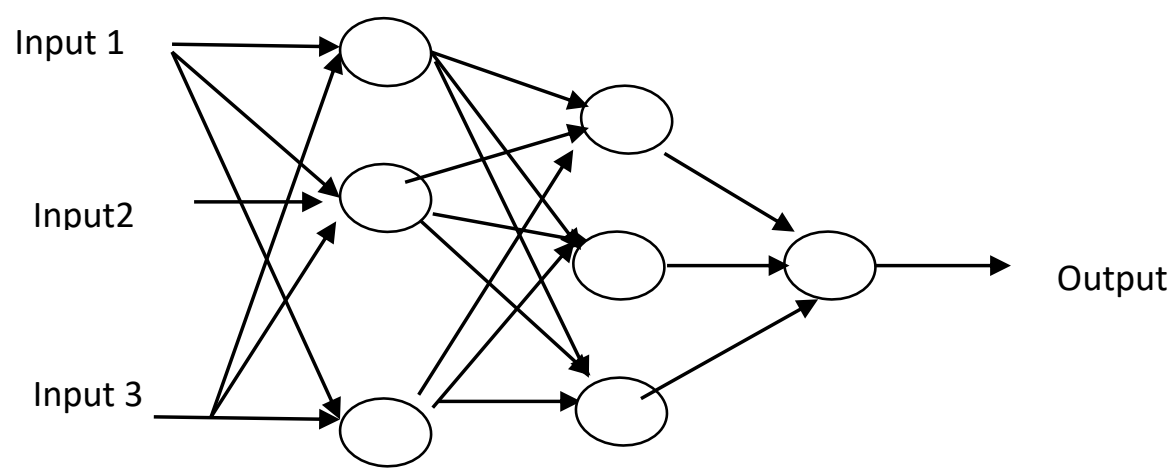

Figure 1. Multi-layer Perceptron Model

The rest of the paper is organized as follows: in Section 2, system model of MC CDMA is described. Section 3 provides description of MAI mitigation techniques such as PIC with ML equalizer and PIC with ANN. In section 4 simulation of the results and analysis are done and finally section 5 concludes the paper.

\section{System Model}

\subsection{Transmitter Section of MC-CDMA}

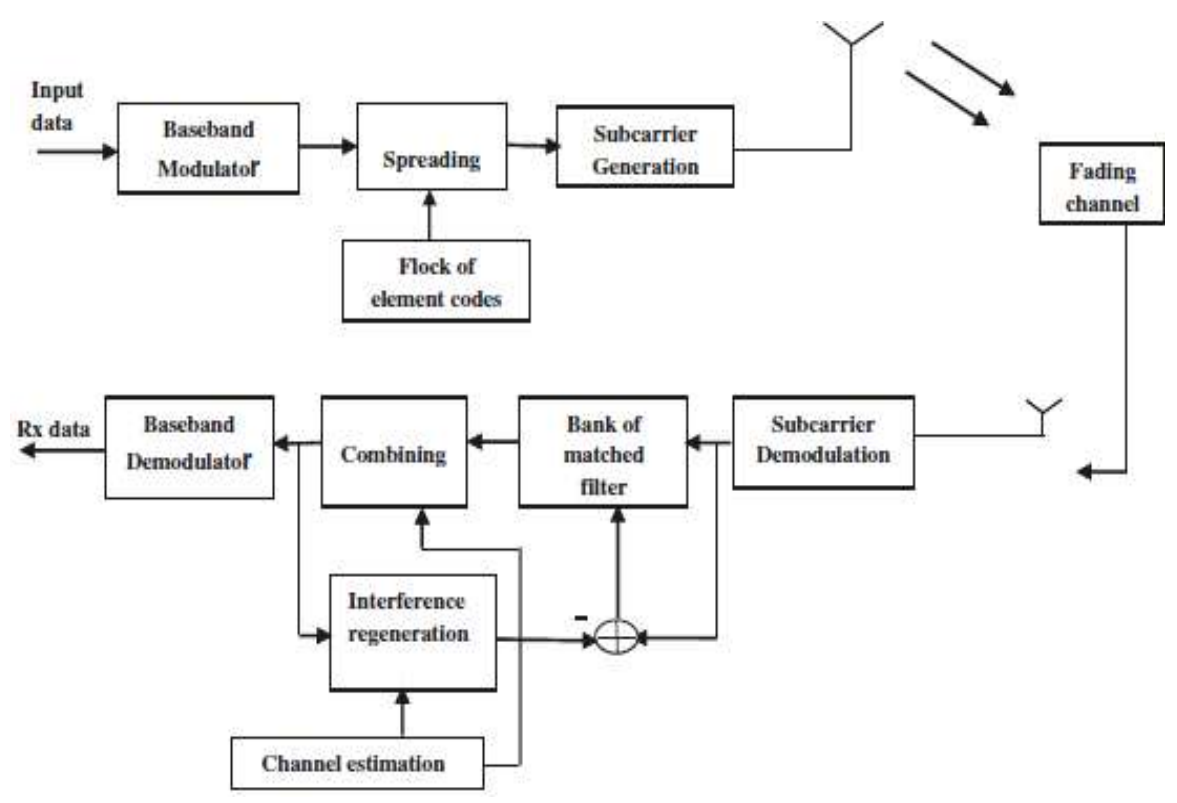

Figure 2. Block Diagram of MC-CDMA

Figure 2 describes the overall MC-CDMA communication system. At transmitter side in MC-CDMA if the numbers of users are $K$, after performing baseband modulation, $\mathrm{k}^{\text {th }}$ user information is represented as $a_{k}(t)$. Thus modulated symbols are spread with group of element codes represented as: $\mathrm{C}^{(\mathrm{K})}=\left[C_{1}^{(k)}, C_{2}^{(k)}\right.$ $\left.C_{N}^{(k)}\right]^{T}$,
(1)Where, $\mathrm{k}$ is $1,2, \ldots . \mathrm{K}$ and $\mathrm{n}=1,2, \ldots \ldots \mathrm{N}$.

In the above equation $\mathrm{C}^{(\mathrm{k})}$ represents code and $C_{n}^{(k)}$ represents corresponding element code respectively. $\mathrm{K}$ in this equation shows number of users and flock size $\mathrm{N}$ is the number of element codes used by the same user. 
In MC-CDMA the data matrix of user $\mathrm{k}$ after spread is represented as:

$$
s(k)=\left[\begin{array}{cccc}
a_{k 1} c_{1}^{(k)} & a_{k 2} c_{1}^{(k)} & \ldots & a_{k N} c_{1}^{(k)} \\
a_{k 1} c_{2}^{(k)} & a_{k 2} c_{1}^{(k)} & \ldots & a_{k N} c_{2}^{(k)} \\
a_{k 1} c_{3}^{(k)} & a_{k 2} c_{3}^{(k)} & \ldots & a_{k N} c_{3}^{(k)} \\
& \cdot & \\
& \cdot & \\
& & \\
a_{k 1} c_{N}^{(k)} & a_{k 2} c_{N}^{(k)} & \ldots & a_{k N} c_{N}^{(k)}
\end{array}\right] \ldots
$$

In the above matrix $\left[a_{k 1}, a_{k 2} \ldots ., a_{k N}\right]$ represents the data which corresponds to the user $\mathrm{k}$ and user's data has been sent along the multiple carriers to the receiver side. Equation 2 represents the reason of various side lobes at the time of autocorrelation among individual element codes. For two different codes sum of correlation between $\mathrm{N}$ elements sequences is zero. The data $\mathrm{a}_{\mathrm{k}}(\mathrm{t})$ spread with $\mathrm{N}$ element codes followed by subcarrier modulation is denoted as

$$
S^{(k)}(t)=\sqrt{p} \sum_{n=1}^{N} a_{k}(t) C_{n}^{(k)}(t) e^{j \emptyset n}
$$

Where, $\mathrm{P}=\mathrm{E}_{\mathrm{b}} /\left(\mathrm{NT}_{\mathrm{b}}\right)$ and $\mathrm{T}_{\mathrm{b}}=\mathrm{L}_{\mathrm{c}} \mathrm{T}_{\mathrm{c}} . \mathrm{T}_{\mathrm{b}}$ is the chip interval and $e^{j \emptyset n}$ represents the subcarrier appointed to $\mathrm{n}^{\text {th }}$ element code. $C_{n}^{(k)}(t)$ Is the spreading waveform of user $\mathrm{k}$ which can be defined as,

$$
C_{n}^{(k)}(t)=\sum_{m=0}^{l c-1} C_{n, m}^{(k)} \Phi(t-m T c), \quad 0<t \leq T_{\mathrm{b},}
$$

Equation (4) describes $C_{n, m}^{(k)}$ as the value of the chip of the spreading sequence assigned to the user k. $\Phi(t)$ is the rectangular shape of the chip limited waveform. Subcarrier modulation is performed on signal of $\mathrm{K}$ users and then signals are sent over the frequency selective fading channel synchronously. Since bandwidth of each sub carrier is supposed to be less than the bandwidth of the whole system a downlink transmission is considered.

\subsection{Receiver Section of MC-CDMA}

Consider desired user of interest is $l$, and demodulated signal received from transmitter subcarrier $n$ in a mobile station as:

$$
r_{n}^{(v)}(t)=h_{n}^{(k)} \sum_{k=1}^{k} s_{n}^{(k)}(t)+n_{n}(t)
$$

Where, $h_{n}^{(l)}=\left|h_{n}^{(k)}\right| e^{j \phi_{n}^{(l)}}$ is channel coeffeicnt, and $s_{n}^{(k)}(t)$ is spreaded data of $\mathrm{n}^{\text {th }}$ element code of a user $\mathrm{k}, n_{n}(t)$ is gaussian noise.

In MC-CDMA system carrier demodulated signals of individual user have been transmitted through $\mathrm{N}$ chip-matched filters corresponding to $\mathrm{N}$ element sequences. $y_{n}^{(k)}(t)$ represents the chip matched filter output for element sequence $c_{n}^{(t)}$ at the reciever side of desired user 1 , thus $\mathrm{n}^{\text {th }}$ element sequence under perfect carrier code in matched filter output and bit synchronization is decribed as:

$$
y_{n}^{(t)}(t)=\int_{0}^{T} T_{n}^{(t)}(t) c_{n}^{(t)}(t) d t .
$$

By substituting resulting equation become as: 


$$
y_{n}^{(l)}(t)=h_{n}^{(1)} \sqrt{p} \sum_{k=1}^{K} b_{k}(t) \rho_{k_{k} l_{k} 0}^{(n)}+I_{n}
$$

$I_{n}$ in the equation (7) shows the sampled additive white Gaussian noise with zero mean and varaiance can be expressed as:

$$
I_{n}=\int_{0}^{T} n_{n}(t) c_{n}^{(l)}(t) d t
$$

For the desired user outputs from $\mathrm{N}$ chip matched filters are combined. Now, the signals received through $\mathrm{N}$ subcarriers results into $\mathrm{N}$-branch diversity, where each branch is the copy of source data spread by the $n^{\text {th }}$ element sequence. Therefore, for achieving ideal correlation of received signal an appropriate combining scheme must be used to get frequency diversity gain.

\section{MAI Reduction Using Interference Cancellation Technique}

3.2.1. Maximum Likelihood Equalizer for Interference Cancellation: The output $y_{n}^{(l)}(t)$ of matched filter is given as input to maximum likelihood equalizer as it is considered to be optimal for error detection and correction. With the use of equalizer Bit Error Rate (BER) improves and hence it provides better signal to noise ratio. It can be represented as:

The Maximum Likelihood Receiver aims at finding $\mathrm{x}$ to minimize,

$$
\begin{gathered}
\mathrm{J}=|y-H x|^{2} \ldots \ldots \ldots \ldots \ldots \ldots \ldots \ldots \\
j=\left|\left[\begin{array}{l}
y 1 \\
y 2
\end{array}\right]-\left[\begin{array}{ll}
h 11 & h 12 \\
h 21 & h 22
\end{array}\right]\left[\begin{array}{l}
x 1 \\
x 2
\end{array}\right]\right|^{2} . . .
\end{gathered}
$$

Since QPSK modulation is performed, possible values of $\mathrm{x}_{1}$ are +1 and -1 and similarly +1 and -1 values are considered for $\mathrm{x}_{2}$. Hence for finding the maximum likelihood solution, we must find minimum of all the four combination of $x_{1}$ and $x_{2}$ [14].

$$
\begin{aligned}
& j_{(+1,+1)}=\left|\left[\begin{array}{l}
y 1 \\
y 2
\end{array}\right]-\left[\begin{array}{ll}
h 1,1 & h 1,2 \\
h 2,1 & h 2,2
\end{array}\right]\left[\begin{array}{l}
+1 \\
+1
\end{array}\right]\right|^{2} \cdot \\
& j_{\left(+1_{0}-1\right)}=\left|\left[\begin{array}{l}
y 1 \\
y 2
\end{array}\right]-\left[\begin{array}{ll}
h 1,1 & h 1,2 \\
h 2,1 & h 2,2
\end{array}\right]\left[\begin{array}{l}
+1 \\
-1
\end{array}\right]\right|^{2} . \\
& j_{(-1,+1)}=\left|\left[\begin{array}{l}
y 1 \\
y 2
\end{array}\right]-\left[\begin{array}{ll}
h 1,1 & h 1,2 \\
h 2,1 & h 2,2
\end{array}\right]\left[\begin{array}{l}
-1 \\
+1
\end{array}\right]\right|^{2} \ldots \\
& j_{(-1,-1)}=\left|\left[\begin{array}{l}
y 1 \\
y 2
\end{array}\right]-\left[\begin{array}{ll}
h 1,1 & h 1,2 \\
h 2,1 & h 2,2
\end{array}\right]\left[\begin{array}{l}
-1 \\
-1
\end{array}\right]\right|^{2} \ldots \ldots
\end{aligned}
$$

Transmit symbol's estimate is selected on the basis of minimum value from the above values

If the minimum value is $j_{(+1,+1)}=[11]$, 
If the minimum value is $j_{(+1,-1)}=\left[\begin{array}{ll}1 & 0\end{array}\right]$,

If the minimum value is $j_{(-1,+1)}=\left[\begin{array}{ll}0 & 1\end{array}\right]$,

If the minimum value is $j_{(-1,-1)}=\left[\begin{array}{ll}0 & 0\end{array}\right]$.

3.2.2. MRC- based Parallel Interference Cancellation: In MC-CDMA, PIC is applied to $\mathrm{N}$ subcarriers. The main reason of applying PIC is to reconstruct the signal suffering with MAI and recovering the useful signal from the desired user. PIC is employed in two steps. At first, the initial assessment of data due to interfering users is performed using equation (16) with suitable combining method ML equalizer. PIC is applied using equation (15) for regeneration and cancelling MAI existing in the received signal. In the second step, the interference-cancelled signal is combined using Maximal Ratio Combining (MRC).

For subcarrier n, signal after interference cancellation can be written as,

$$
r_{p i c, n(t)=r_{n}}(t)-h_{n \sqrt{p}} \sum_{k=1 . k \neq l}^{K} J^{(k)}(i) c_{n}^{(k)}(t)
$$

Above $r_{n}(t), n \in\{1,2 \ldots N\}$, denotes the received signal for sub-carrier $n$ of all the $K$ users in the system. $\mathrm{J}^{\wedge}(\mathrm{k})(\mathrm{i})$ is evaluated based on output of maximum likelihood equalizer, which represents the interference at the output of chip-matched filter because of interfering $\mathrm{k}$ users.

After interference cancellation, next step is to de-spread the signals from $\mathrm{N}$ subcarrier using same complementary code as used for spreading for desired user $l$ and then perform combining to give the estimated data as:

$$
b_{\text {pic }}^{(i)}(i)=\sum_{n=1}^{N} w_{n}^{(l)} \int_{0}^{T b} r_{\text {pic, } n}(t+i T b) c_{n}^{(l)}(t) d t
$$

The data signal of desired user $l$ after interference estimation using a estimation based on output of ML receiver and MRC combining is given by

$$
b_{\text {pic }}^{(l)}(i)=L_{C} \sqrt{P} \sum_{n=1}^{N}\left|h_{n}^{(n)}\right|^{2} b^{(l)}(i)+j_{\text {pic }}+N_{\text {pic }}
$$

Here $\mathrm{N}_{\text {pic }}$ denotes noise and $\mathrm{J}_{\text {pic }}$ denotes residual MAI represented as

$$
j_{\text {pic }}=\sqrt{p} \sum_{k=1_{l} k \neq l}^{K}\left(b^{(k)}-j^{(k)}\right) \sum_{N=1}^{N}\left|h_{n}^{(l)}\right|^{2} \rho_{k_{k} l, 0}^{(n)}
$$

It is observed from equation (18), the performance of MC-CDMA depends on the output of ML receiver and the correlation properties of each element sequences. In addition, the accuracy of the estimated MAI $\mathbf{J}^{\wedge}{ }^{(\mathrm{k})}(\mathrm{i})$ also depends on channel state information available at the receiver.

3.2.3. MC-CDMA Receiver using PIC-ANN: Multi-Layer PerceptronS (MLPS) are made up of input, hidden and output layers. There are fed forward connections among neurons. Input layer act as buffer to disperse the input signal to the neuron of hidden layer. In the neurons many activation functions are used. The weights attached to the neuron are adjusted according to learning algorithm to get the desired output. Hence, for the optimization of output of parallel interference cancellation, a new feed forward back propagation neural network with three inputs and one output can be used. Network training is performed with gradient descent back propagation function i.e., "traingd", which is a network training function that updates weight and bias value according to gradient descent. Similarly, gradient descent weight and bias learning function i.e "learngd" is used. In the hidden layer tangent sigmoid activation function i.e., "tansig" is used and at the output layer pure linear activation function i.e "purlin" is used. 
The signal after passing through Nakagami channel is received by the detection scheme, which consists of the Fast Fourier Transform (FFT) block, ML Equalizer, parallel interference cancellation and Neural Networks (NN) block [15-18]. For each user the spreading code of size $N$ is assigned. The $K \times N$ dimensional received signal is fed to ML equalizer. The $K \times N$ dimensional channel matrix $H$ is another input of the ML equalizer.

$$
\mathrm{H}=\left[\begin{array}{cccc}
h 11 & h 21 & \cdots & h k 1 \\
h 12 & h 22 & \cdots & \\
\vdots & \vdots & \ddots & \vdots \\
h 1 n & h 2 n & \cdots & h k n
\end{array}\right]
$$

The outputs of parallel interference cancellation block are taken as inputs for proposed Neural Networks (NN) receiver. The number of input, hidden and output nodes of the Neural Network model is chosen according to number of users in the MC- CDMA system.

\section{Simulation Analysis and Results}

In this section, simulation results are presented for evaluating the execution of MCCDMA system with different multi user detection techniques. Here for modulation of each sub carrier, a BPSK modulation technique is used. For modeling each sub carrier Nakagami flat fading channel is considered. It is believed that Channel State Information (CSI) is given at the reception end. The simulations are performed with complimentary codes.

\subsection{BER Analysis of MC-CDMA with PIC}

In the first stage, the initial data estimates of all interfering users is obtained using ML Equalizers and PIC is applied to regenerate and cancel MAI occurring in the received signal. In the second stage the interference cancelled signal is combined using MRC forming ML-PIC-MRC. Following steps are followed for simulation purpose.

\section{At Tx section}

Step 1: Initially, generate complementary code.

Step 2: Generate information bits and appended to the signal.

Step 3: Perform modulation on the signal.

Step 4: Execute Inverse FFT operation on the modulated signal.

Step 5: Add cyclic prefix bits to the generated signal from the previous stage.

Step 6: Locate the Nakagami effect on the signal.

\section{At Rx Section}

Step 1: At the receiving side, originally cyclic prefix bits have removed from the signal.

Step 2: Perform FFT operation on the signal.

Step 3: Then perform equalization on the signal to generate output signal.

Step 4: To regenerate and cancel MAI, apply parallel Interface Cancellation onto signal.

Step 5: Finally, calculate the error in the signal for estimation of performance of the technique. 
Here nakagami- $\mathrm{m}$ channel is considered, where value of fading parameter, $\mathrm{m}=0.5$. Here, 4 users are considered and length of element code, $L_{c}=32$. After simulation it is observed that at $6 \mathrm{~dB}$ SNR, BER is 0.0028 as shown in Figure 3.

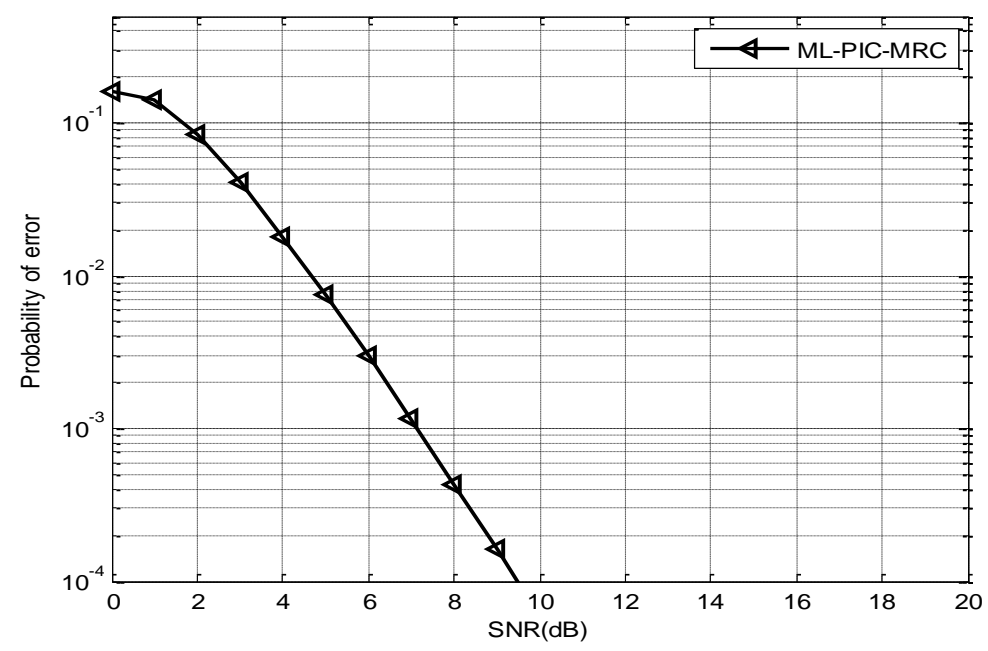

Figure 3. Performance Analysis of Parallel Interference Cancellation using ML Equalizer

\subsection{BER Analysis OF MC-CDMA with PIC-ANN}

In this section, the BER performance of MC-CDMA using ML- PIC with ANN is analyzed. Here ANN is used for the optimizing the output of PIC. Figure 4 shows the performance improvement achieved by using ML-PIC-ANN. Output is evaluated after training and testing. Here 100 epochs are taken into consideration.

Figure 4 also shows an improved performance when Artificial Neural Network with Parallel Interference Cancellation is used under frequency selective channels using same parameters as ML-PIC-MRC. Further it can be observed that at 6dB SNR the output of MC-CDMA with ML-PIC-MRC is 0.0028 and with ML-PIC-ANN is 0.0015 . This proves that ML-PIC-ANN performs better than ML-PIC-MRC. Hence the result has been improved by using Artificial Neural Network with Parallel Interference Cancellation.

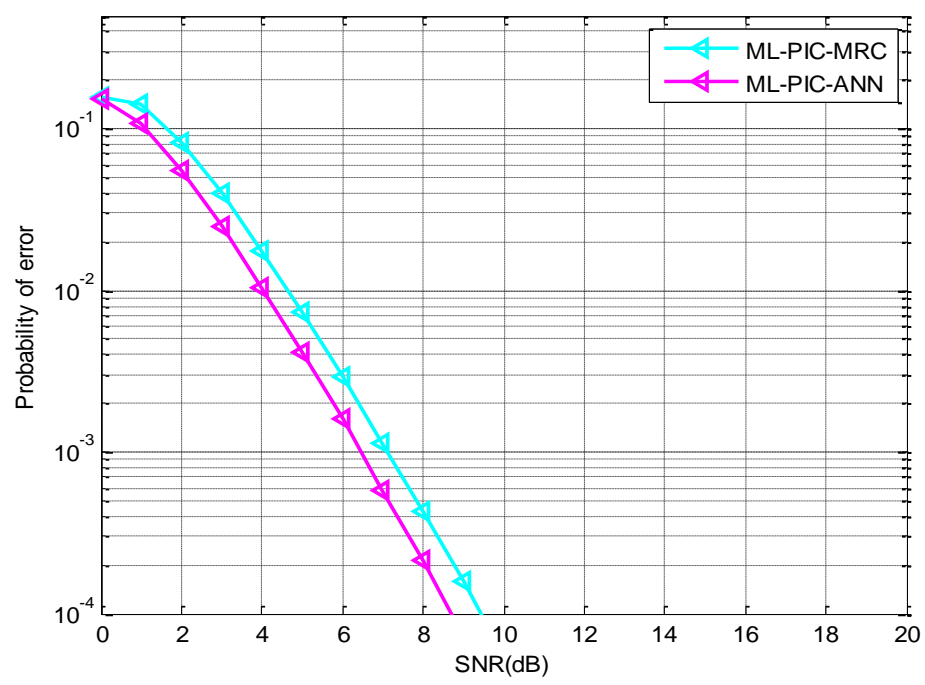

Figure 4. Performance Comparisons between PIC and PIC-ANN 


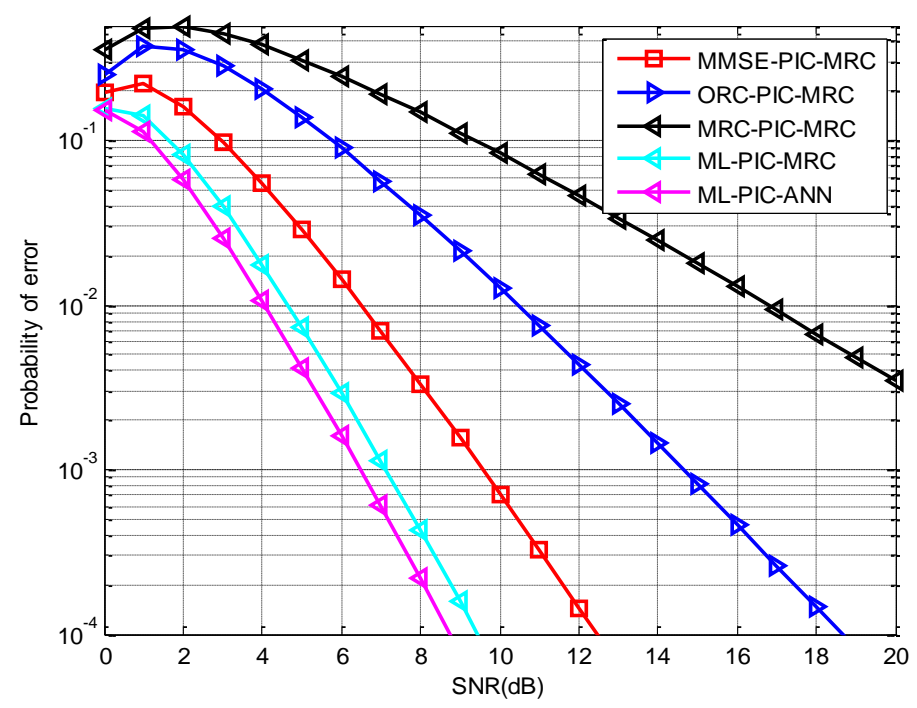

Figure 5. Comparison of Difference Parallel Interference Techniques

The existing technique provides better result when PIC is applied to combining schemes such as MMSE, MRC and ORC. It has proved that MMSE-PIC with MRC combining gives improved results for mitigating MAI in comparison to ORC-PIC and MRC-PIC with BER of 0.0144. This BER is higher than the BER of proposed technique as shown in Figure 5. Hence, it can be analyzed that the results have been improved by using ML equalization instead of different combining scheme. Further, the BER can be reduced by combining PIC with ANN.

\section{Conclusion and Future Work}

In this work the effect of MAI is detected and reduced by using proposed technique of artificial neural network based Parallel interference cancellation technique to the MCCDMA communication system. Simulation performed on different interference cancellation techniques such as Parallel interference cancellation with ML equalization (ML-PIC-MRC) and neural network based parallel interference cancellation (ML-PICANN) in MC-CDMA systems. Performance is evaluated in terms of BER versus SNR. After result comparison is done between the proposed technique ML-PIC-ANN with and ML-PIC. It is concluded that when neural network is combined with ML-PIC, it optimizes the output of ML-PIC cancellation and hence reduces the BER. BER of ML-PIC-MRC and ML-PIC-ANN is 0.0028 and 0.0015 respectively. Further this proposed technique is compared with the existing result of PIC with different combining schemes. The results clearly indicate that bit error rate is reduced in comparison to other existing results. In future, experiments can be done by using different type of fading channels such as Rican fading channel, Rayleigh fading channel etc. and different modulation schemes.

\section{Conflict of Interest}

The author(s) declare(s) that there is no conflict of interest regarding the publication of this paper.

\section{References}

[1] K. S. Gilhousen, I. M. Jacobs, R Padovani and A Viterbi, "On the capacity of cellular CDMA systems", IEEE Trans. Commun., vol. 40, no. 2, (1991), pp. 303-312. 
[2] S Moshavi, "Multiuser detection for DS-CDMA communications", IEEE Commun. Mag., vol. 34, (1996), pp. 124-136.

[3] H. H. Chen, J. F. Yeh and Suehiro, "A multicarrier CDMA architecture based on orthogonal complementary codes for new generations of wideband wireless".

[4] M. E. Magana and T. Rajatasereekul, "Complete complementary orthogonal (CCO) code based CDMA using natural mapping QAM constellations", Wireless Pers. Commun., vol. 38, no. 4, (2006), pp. 435442.

[5] L. Lu and V. Dubey, "Performance of a complete complementary code based spread-time CDMA system in a fading channel”, IEEE Trans. Vehicular Technol., vol. 57, no. 1, (2008), pp. 250-259.

[6] H. H. Chen, "The next generation CDMA technologies", John Wiley and sons, NJ, USA, (2007).

[7] H. V. Poor and S. Verdu, "Single-user detectors for multiuser channels", IEEE Transactions on Communications, vol. 36, (1998), pp. 50-60.

[8] Z. Xie, R. Short and C. Rushforth, "A family of Suboptimum Detectors for Coherent Multiuser Communications", IEEE Journal on selected areas of Communication, (1990), pp. 683-690.

[9] Y. Li and R. Steele, "Serial interference cancellation method for CDMA", IEEE Electronics letters, vol. 30, (1994), pp. 1581-1583.

[10] W. Hesse and T. Schulz, "Parallel Interference Cancellation (PIC) applied to an asynchronous MCCDMA system", Proceedings of Vehicular Technology Conference (VTC), IEEE, vol. 2, (2002), pp 689-693.

[11] Y. Isik and N. Taspinar, "Multiuser detection with Neural Network and PIC in CDMA systems for AWGN and Rayleigh fading asynchronous channels", Wireless Personal Communications, vol. 43, no. 4, (2007), pp. 1185-94.

[12] K. P. Bagadi and S. Das, "Multiuser detection in SDMA-OFDM wireless communication system using complex multilayer perceptron Neural Network", Wireless Personal Communications, vol. 77, no. 1, (2014), pp. 21-39.

[13] W. H. Meng, S. Y. Sun, H. H. Chen, "Multiuser interference cancellation in complementary coded CDMA with diversity gain", IEEE Wireless Commun. Lett., vol. 2, no. 3, (2013), pp. 303-306.

[14] S. Y. Sun, W. H. Meng and H. H. Chen, "Uplink pre-equalization for CC-CDMA systems under frequency selective fading”, Proc. of IEEE ICC, (2013), pp. 5317-5321.

[15] S. Hara and R. Prasad, "Overview of multicarrier CDMA", IEEE Commun. Mag., vol. 39, no. 12, (1997), pp. 126-133.

[16] S. Verdu, "Multiuser detection", Cambridge University Press, UK., (1998).

[17] B. A. Al-fuhaidi, H. E. A. Hassan, M. M. Salah and S. S. Alagooz, "Parallel interference cancellation with different linear equalization and Rake receiver for the downlink MC-CDMA systems", IET Commun., vol. 6, no. 15, (2012), pp. 2351-2360.

[18] V. A. Aalo, "Performance of maximal-ratio diversity system in a correlated Nakagami-faded environment", IEEE Trans. Commun., vol. 43, no. 8, (1995), pp. 2360-2369.

[19] J. G. Proakis, "Digital communications", 4th edn., McGraw Hill Inc, NY, (2001).

[20] I. S. Gradshteyn and I. M. Ryzhik, "Table of integrals, series and products", 6th edn., Academic, San Diego, (2000). 